\title{
The Synthesis of Diazinon with The Catalysis of Ionic Liquid under The Condition of Microwave
}

\author{
Lin $\mathrm{HU}$ \\ Institute of Applied Chemistry \\ East China JiaoTong University \\ Nanchang, 330013, PR China \\ e-mail: hulin21@hotmail.com \\ Xiaoqin ZHOU* \\ Institute of Applied Chemistry \\ East China JiaoTong University \\ Nanchang, 330013, PR China \\ e-mail: 1061508795@qq.com \\ Jingqing LI \\ Institute of Applied Chemistry \\ East China JiaoTong University \\ Nanchang, 330013, PR China \\ e-mail: 1604815540@qq.com
}

\author{
Yegui WANG \\ Institute of Applied Chemistry \\ East China JiaoTong University \\ Nanchang, 330013, PR China \\ e-mail: 819086343@qq.com
}

\author{
Juan TANG \\ Institute of Applied Chemistry \\ East China JiaoTong University \\ Nanchang, 330013, PR China \\ e-mail: 2249113892@qq.com \\ Kuijiang WONG \\ Institute of Applied Chemistry \\ East China JiaoTong University \\ Nanchang, 330013, PR China \\ e-mail: 794138148@qq.com
}

\author{
Wenyuan XU \\ Institute of Applied Chemistry \\ East China JiaoTong University \\ Nanchang, 330013, PR China \\ e-mail: Xwy1027@sina.com
}

\begin{abstract}
A novel synthetic methods for the efficient and environmentally-friendly production will provide useful inspiration. This report presents a novel method for microwave-assisted synthesis of diazinon with different organic solvents, microwave heating power, heating time, catalysts. The optimal conditions for this synthesis are: $750 \mathrm{~W}$ for microwave power, $12 \mathrm{~min}$. for heating time, and $0.02 \%$ for mole ratio of catalyst DMAP to $O$, O-diethyl thiophosphoryl chloride and 1, 2-dichloroethane for the best solvent, which can lead the yield of diazinon to $67.86 \%$. When adding repeatedly fresh 2-isopropyl-6-methyl-4-hydroxy pyrimidine for enough times, the yield of diazinon for $\mathbf{O}$, O-diethyl phosphoryl chloride can be as high as $\mathbf{8 6 . 5 4 \%}$. With an amazing chemical reaction rate and less waste water discharge, microwaveassisted synthesis is a promising method for green synthesis.
\end{abstract}

Keywords-diazinon; optimization of reaction conditions; microwave-assisted synthesis

\section{INTRODUCTION}

As an environmentally benign technology with wide application, microwave synthesis has the advantages of homogeneous volumetric heating, and high reaction rate compared with other physical and chemical methods [1, 2].
Diazinon is a kind of important biochemical compounds. At present, it has developed a variety of synthetic routes of diazinon, and the most representative one is isobutyronitrile synthetic route, which takes isobutyronitrile, methyl acetoacetate, and ethyl chloride as main raw materials, synthesizes diazinon through the amidine, cyclization and condensation reaction [3, 4]. Each step reaction must be carried out under anhydrous conditions and supplemented with appropriate catalysts. In the final step of condensation reaction, if we use the phase transfer method, the reaction rate is fast, the unit productivity is high and the cost is relatively low [5-8]. Because of the reaction occurring at the interface between water phase and oil phase, $\mathrm{O}$, O-diethyl thiophosphoryl chloride will be consumed due to hydrolysis, resulting in low yield and the purity of product is also reduced by mixing with some impurities. In particular, the process of synthesis is accompanied with producing a large amount of wastewater, which contains organic phosphorus waste water and is difficult to be biochemically degraded and it will bring about considerable pressure on the environment $[9,10]$. Nowadays, environmental protection is tightening, with organic solvent as media, at the presence of acid binding agents and catalyst, the synthesis of diazinon through condensation reaction has become the mainstream 
of industrial production. Hu Zhongfu[11] adopts the conventional heating method, the synthetic route takes isobutyronitrile as raw materials, after amidine, cyclization and condensation, the total yield reaches $73.7 \%$ and the reaction time is about $4 \mathrm{~h}$. By orthogonal experiment, Liu Jinhong [12] uses suitable solvent and water carrier, the condensation reaction time is shortened to $6 \mathrm{~h}$ and the content of sulfotepp byproduct is less than $0.13 \%$.

Microwave assisted organic solvent combined with the advantages of organic solvent and microwave technology, the reaction rate is fast, the yield of the product is high, the selectivity is good and the post-treatment is simple. Organic solvent can be recycled for many times, and it can be applied in various types of organic synthesis. By comparing the synthetic yield under different conditions of microwave assisted synthesis, the effects of the factors such as the heating time, the power of microwave radiation, the type of catalyst and solvent on the yield were optimized.

Through the experiment, it found the optimum reaction conditions of microwave assisted synthesis of diazinon, which provided experimental basis for improving the conversion ratio of $\mathrm{O}$, O-diethyl thiophosphoryl chloride in industrial production, reducing the discharge of waste water and environmental pollution.

\section{EXPERIMENTAL}

\section{A. Instruments and Reagents}

Microwave oven (MM823EA6-PS) was purchased from Qingdao Makewave Co., Ltd. Electronic balance (JY203) was purchased from Shanghai Puchun Measuring Instrument Co., Ltd. GC-950 was supplied by Shanghai Haixin Chromatographic Instrument Co, Ltd. High pressed test tube(FD-004) was purchased from Golden Lake Fidelity Machinery Co., Ltd.

2-isopropyl-6-methyl-4-hydroxy pyrimidine(95.0\%) were supplied by Nantong Jiangshan Agrochemical Co., Ltd. The prototype diazinon was supplied by AccuStandard. Other drugs and reagents are analytically pure and can be used directly; the anhydrous solvent is treated according to the standard method.

\section{B. Synthesis Principle}

The 2-isopropyl-6-methyl-4-hydroxy pyrimidine reacted with $\mathrm{NaOH}$ to produce sodium 2-isopropyl-6methyl-4-hydroxy pyrimidine, which was shown in scheme1.

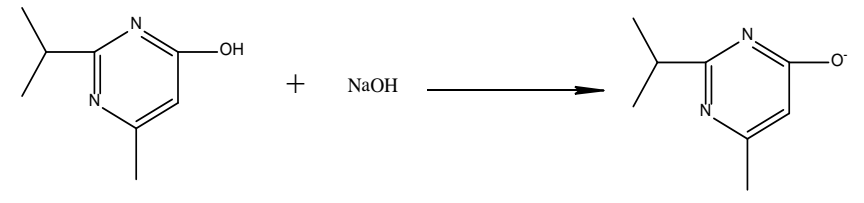

Scheme 1

Sodium 2-isopropyl-6-methyl-4-hydroxy pyrimidine reacted with $\mathrm{O}$, O-diethyl phosphoryl chloride under different conditions to produce diazinon, which was shown in scheme 2[13-15].

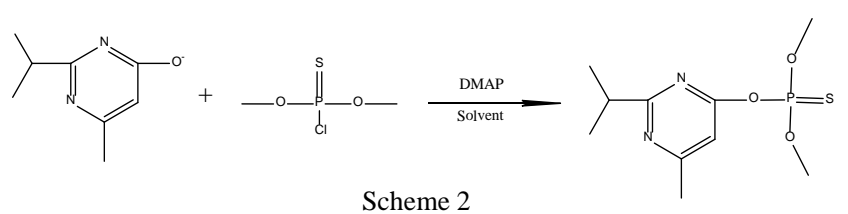

\section{Experimental Operation}

1) The preparation of sodium 2-isopropyl-6-methyl-4hydroxy pyrimidine[16]: Put 2-isopropyl-6-methyl-4hydroxy pyrimidine (95\%, $2 \mathrm{~mol}) 320.42 \mathrm{~g}$ into $1000 \mathrm{~mL}$ beaker, then added the distilled water $500 \mathrm{~mL}$ and $\mathrm{NaOH}$ $88 \mathrm{~g}$ into the beaker slowly and stirred until the 2-isopropyl6-methyl-4-hydroxy pyrimidine was dissolved. We could obtain the aqueous solution of sodium 2-isopropyl-6methyl-4-hydroxy pyrimidine when the aqueous solution was clear and transparent. Put the aqueous solution into $1000 \mathrm{~mL}$ round flask, dehydrated $8 \mathrm{~h}$ under heating and decompression, when appeared white solid precipitations, removed and crushed it, then continued to place it in the oven at $100^{\circ} \mathrm{C}$ until sodium 2-isopropyl-6-methyl-4-hydroxy pyrimidine (98.2\%) was completely dry.

2) The diazinon synthesis[17]: Took the dried sodium 2-isopropyl-6-methyl-4-hydroxy pyrimidine $2.325 \mathrm{~g}$ (98.2\%, 0.015 mol), DMAP $0.001 \mathrm{~g}$ (DMAP is $0.02 \%$ mole ratio with 2-isopropyl-6-methyl-4-hydroxy pyrimidine), the solvent 15g, O,O-diethyl thiophosphoryl chloride $3.445 \mathrm{~g}$ (98.5\%, $0.018 \mathrm{~mol})$, and put them in $90 \mathrm{~mL}$ high pressed test tube, then stayed in microwave oven (MM823EA6-P), adjusted the position of high pressed test tube to a right place. Set different powers, heating mode was set to work 3 min and stop $5 \mathrm{~min}$, and then work $3 \mathrm{~min}$ and stop $5 \mathrm{~min}$, did this again and again until the set time. After the reaction, cooled and filtrated the internal reaction liquid and solid, for ionic liquids could be used as reaction solvent, then used ether extraction, and detected content.

\section{Determination of Diazinon by Gas Chromatography}

1) Chromatographic conditions [18]: Chromatographic column, $\phi 3 \times 0.5 \times 1000$ stainless steel column; fillers, $5 \%$ OV$101 /(\mathrm{m} / \mathrm{m}$ ) silanization (HMDS),101white but body (80 100mesh); column temperature, $200^{\circ} \mathrm{C}$ (glass thermometer indicator); detection temperature, $230^{\circ} \mathrm{C}$ (indicating value of the instrument meter); gasification chamber temperature, $240^{\circ} \mathrm{C}$ (indicating value of the instrument meter); carrier gas, the velocity of $\mathrm{N}_{2}$ was $30 \mathrm{~mL} \cdot \mathrm{min}^{-1}$, the velocity of $\mathrm{H}_{2}$ was $40 \mathrm{~mL} \cdot \mathrm{min}^{-1}$, the velocity of $\mathrm{O}_{2}$ was $350 \mathrm{~mL} \cdot \mathrm{min}^{-1}$; sample volume was $0.6 \mu \mathrm{L}$.

2) Measuring steps: i[5,13]. Preparation of internal standard solution: put $0.25 \mathrm{~g}$ diethyl phthalate $(\geq 98.0 \%)$ dissolved in acetone in the $50 \mathrm{~mL}$ volumetric flask, diluted to scale and shook well. The concentration was $5 \mathrm{mg} \cdot \mathrm{mL}^{-1}$.

ii. Preparation of diazinon standard solution: put $0.035 \mathrm{~g}$ standard diazinon in $10 \mathrm{~mL}$ volumetric flask, added $2.0 \mathrm{~mL}$ internal standard solution accurately, diluted to scale with 
ethanol or acetone, shook well then put in the refrigerator to save.

iii. Preparation of the sample solution: put $0.035 \mathrm{~g}$ sample diazinon in the $10 \mathrm{~mL}$ volumetric flask, added $2.0 \mathrm{~mL}$ internal standard solution accurately, diluted to scale with ethanol or acetone.

iv. Selected chromatographic conditions and inject standard solution several injections before the instrument operation was stable. When the ratio of chromatographic response values of adjacent two samples between diazinon and internal standard substance was less than $2 \%$, according to obtained chromatograms from standard and sample, we could determine the average of area or peak height ratio and use the formula to calculate the percentage content of diazinon [14]:

$$
f=\frac{w_{s}}{w_{i}} \times \frac{A_{i}}{A_{s}} \times p
$$

Where: $A_{\mathrm{i}}$ and $A_{\mathrm{s}}$ are average of area or peak height ratio between diazinon and internal standard substance in standard and sample, respectively. $W_{\mathrm{s}}$ and $W_{\mathrm{i}}$ are quantity of standard and sample. $P$ is the percentage of diazinon in standard.

\section{RESUlTS AND DISCUSSION}

\section{A. Effects of Different Organic Solvents on Microwave Assisted Synthesis of Diazinon}

The mole ratio of sodium 2-isopropyl-6-methyl-4hydroxy pyrimidine and O, O-diethyl phosphoryl chloride was $1: 1.2$, the dosage of catalyst DMAP was $0.02 \%$ mole ratio with O, O-diethyl thiophosphoryl chloride, microwave power was $750 \mathrm{~W}$, the heating time was $12 \mathrm{~min}$. Add different kinds of organic solvent with same amount and use gas chromatography to detect the yield of diazinon. The result showed that the 1, 2-dichloroethane was the best solvent in microwave assisted synthesis, which was shown in Fig. 1.

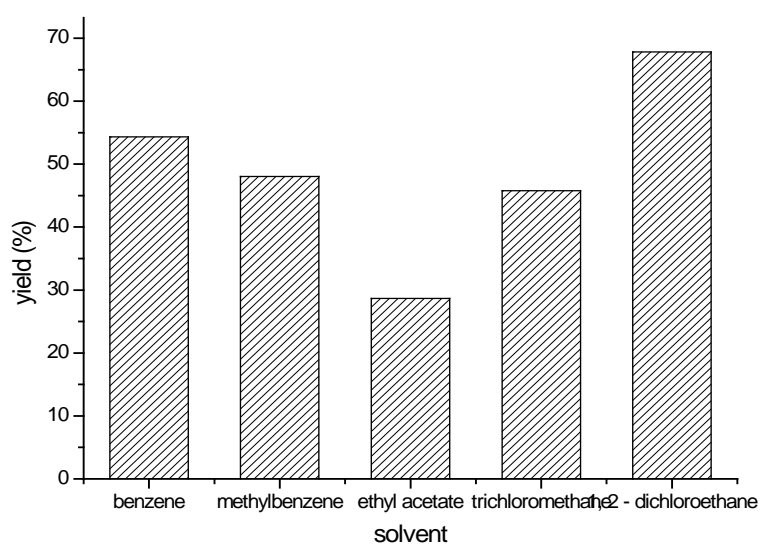

Figure 1. The influence of solvent on the yield of diazinon compared with benzene, methy lbenzene, ethyl acetate, trichlormethane1, and 2dichloroethane.

\section{B. The Effects of Microwave Power on Diazinon Synthesis}

The mole ratio of sodium 2-isopropyl-6-methyl-4hydroxy pyrimidine and O, O-diethyl phosphoryl chloride was $1: 1.2$, the dosage of catalyst DMAP was $0.02 \%$ mole ratio with $\mathrm{O}$, O-diethyl thiophosphoryl chloride, the solvent was 1, 2-dichloroethane, the heating time was $12 \mathrm{~min}$. Use different Microwave power to react. The results showed that the $750 \mathrm{~W}$ was the best power in microwave synthesis, which was shown in Fig. 2.

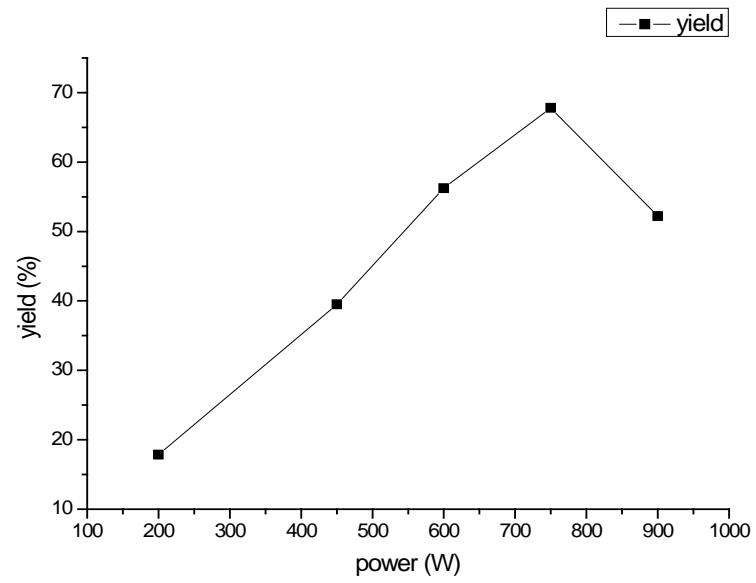

Figure 2. The influence of power on the yield of diazinon.

\section{The Influence of Heating Time on Diazinon Synthesis}

The mole ratio of sodium 2-isopropyl-6-methyl-4hydroxy pyrimidine and O,O-diethyl phosphoryl chloride was $1: 1.2$, the dosage of catalyst DMAP was $0.02 \%$ mole ratio with O,O-diethyl thiophosphoryl chloride, the solvent was 1, 2-dichloroethane, microwave power was set $750 \mathrm{~W}$.Use different microwave heating time to react. The result showed that the $12 \mathrm{~min}$ was the best time in microwave assisted synthesis, which was shown in Fig. 3.

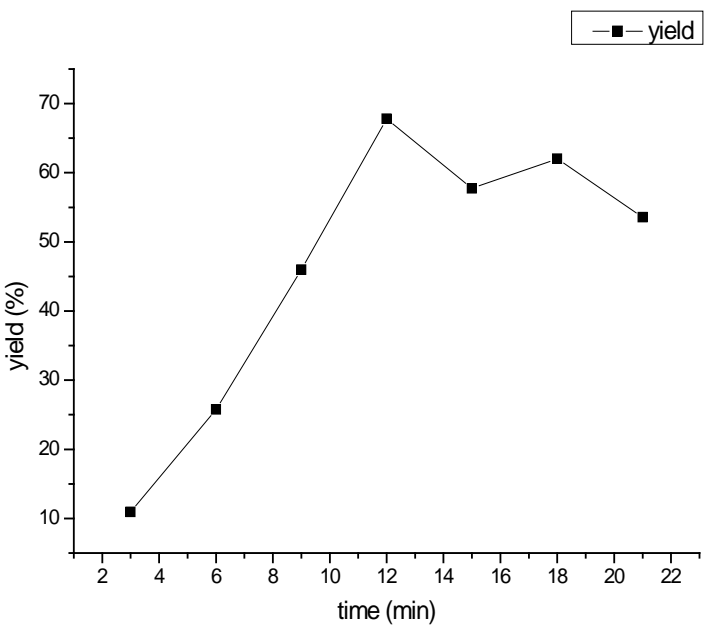

Figure 3. The influence of time on the yield of diazinon. 


\section{The Dosage of Catalyst DMAP Affects on Diazinon Synthesis}

The mole ratio of sodium 2-isopropyl-6-methyl-4hydroxy pyrimidine and $\mathrm{O}$, O-diethyl phosphoryl chloride was $1: 1.2$, the solvent was 1 , 2-dichloroethane, microwave power was set $750 \mathrm{~W}$. The heating time was $12 \mathrm{~min}$. Use different dosages of catalyst DMAP to react. The result showed that $0.02 \%$ mole ratio the dosage of catalyst DMAP with sodium 2-isopropyl-6-methyl-4-hydroxy pyrimidine was the best condition in microwave synthesis, which was shown in Fig. 4.

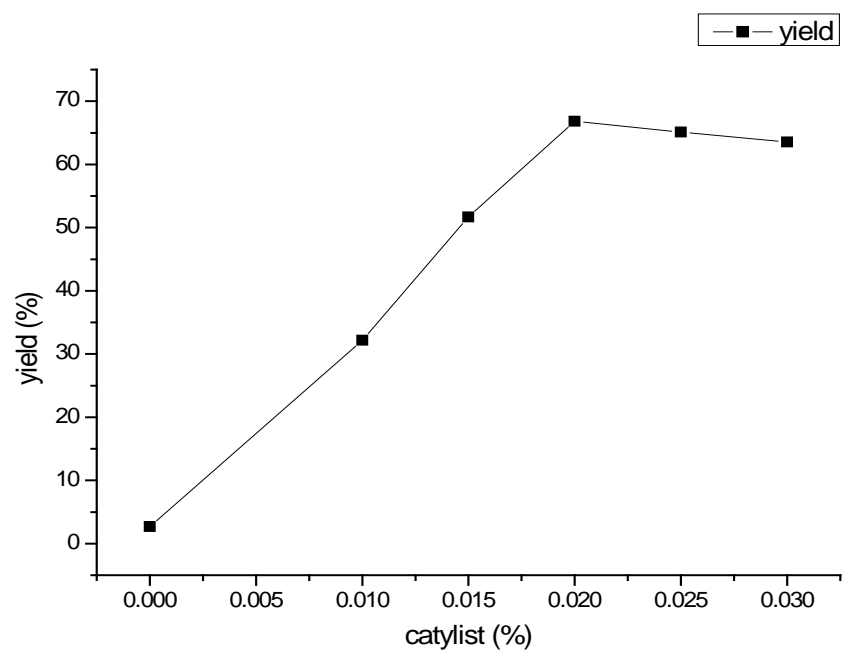

Figure 4. The influence of catalyst DMAP dosage on the yield of diazinon

\section{E. Conversion Rate of O,O-Diethyl Thiophosphoryl Chloride under The Optimized Conditions}

In the previous diazinon synthesis, the yield of diazinon and the O, O-diethyl thiophosphoryl chloride was low, because the sodium 2-isopropyl-6-methyl-4-hydroxy pyrimidine is solid. It reacted with O, O-diethyl phosphoryl chloride to product sodium chloride, which covered on the surface of the sodium 2-isopropyl-6-methyl-4-hydroxy pyrimidine to hinder the further reaction. We had done the following changes of the experiment in order to improve conversion rate. Under the optimal conditions, separate the sodium chloride covered on the surface of sodium 2isopropyl-6-methyl-4-hydroxy pyrimidine every once in a while, then add new sodium 2-isopropyl-6-methyl-4hydroxy pyrimidine to react. After a reaction cycle, filtrated, added new sodium 2-isopropyl-6-methyl-4-hydroxy pyrimidine to react, thus again and again, recorded the yield of every cycle. After separation, sodium 2-isopropyl-6methyl-4-hydroxy pyrimidine can be leached with a little water to remove sodium chloride which was covered on the surface and then be reused.

The mole ratio of sodium 2-isopropyl-6-methyl-4hydroxy pyrimidine and O,O-diethyl phosphoryl chloride was $1: 1.2$, the dosage of catalyst DMAP was $0.02 \%$ mole ratio with O,O-diethyl thiophosphoryl chloride, the solvent was 1, 2-dichloroethane, microwave power was set 750W.
The heating time was $12 \mathrm{~min}$. Interval of $12 \mathrm{~min}$ after heating filter and replace sodium 2-isopropyl-6-methyl-4hydroxy pyrimidine. The concentration of product was detected by gas chromatography. The cumulative conversion of $\mathrm{O}$, O-diethyl thiophosphoryl chloride was shown in Fig.5.

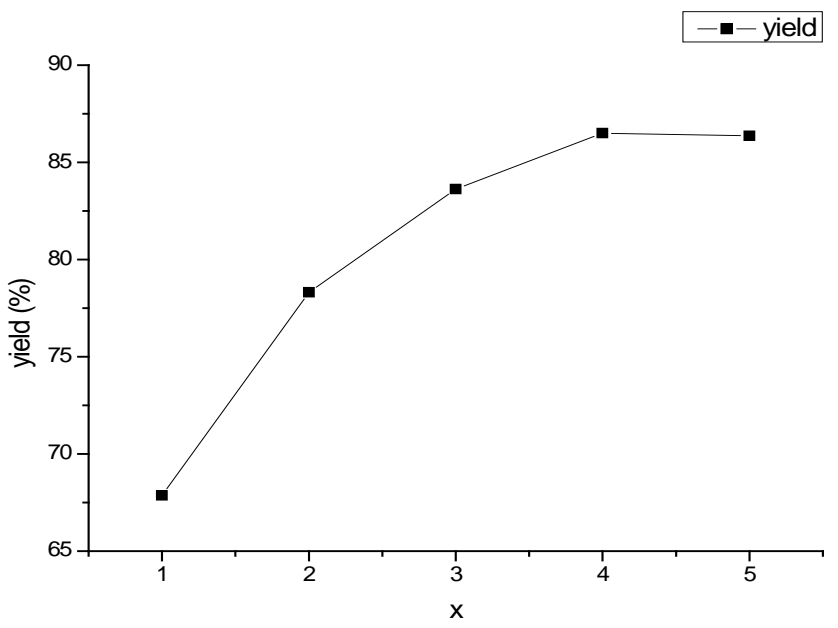

Figure 5. The influence of sodium 2-isopropyl-6-methyl-4-hydroxy pyrimidine cyclic reaction times on the yield of diazinon.

\section{CONCLUSION}

The report adopted the new type of microwave-assisted synthesis and promoted the reaction between organic molecules in a new type of heating method. We have considered the main factors affecting microwave synthesis, such as: 1.the solvent type of reaction system; 2.the microwave power; 3.the microwave heating time; 4.the catalyst in synthesis system. With the different factors changed, the yield of diazinon relatived to the O, O-diethyl phosphoryl chloride also changed accordingly. After an indepth exploration research, we finally obtained the best microwave synthesis scheme.

The optimized conditions: microwave power is $750 \mathrm{~W}$, the reaction time was $12 \mathrm{~min}$, the solvent was 1 , 2dichloroethane, the mole ratio of catalyst DMAP to O, Odiethyl thiophosphoryl chloride was $0.02 \%$, and the yield of diazinon can be increased to $67.86 \%$. After a period of time we separated the sodium chloride covered on sodium 2isopropyl-6-methyl-4-hydroxy pyrimidine surface and added new sodium 2-isopropyl-6-methyl-4-hydroxy pyrimidine to react. After a reaction cycle, then filtrated, added new sodium 2-isopropyl-6-meth-4-hydroxy pyrimidine to react, thus again and again, theyl yield of diazinon for O, O-diethyl phosphoryl chloride could be as high as $86.54 \%$.

In microwave-assisted synthesis, because polar molecules are more favorable for the absorption of microwave radiation than non polar molecules, a certain temperature gradient is generated in the reaction system, which showed chemical behavior different from traditional reaction. With the use of microwave-assisted synthesis, the reaction time can be shortened to 30 minutes while the room 
temperature heating method requires a few hours. Ionic liquids are polar molecules, all of which is composed of ions, which can absorb microwave energy efficiently. It is an excellent solvent and cosolvent in microwave reaction. The reaction in ionic liquid under microwave condition is rapid, the yield is high, the selectivity is good, and the post processing is simple, the ionic liquid can be repeatedly used after simple regeneration. Microwave-assisted ionic liquid phase organic synthesis is a new kind of green chemical synthesis method, which has aroused great interest. The use of this technology will greatly reduce the amount of chemical waste and reaction time in organic synthesis and chemical conversion process, greatly promoting the development of green chemistry and organic synthesis methodology.

\section{ACKNOWLEDGMENT}

The authors gratefully acknowledge funding of this work by the National Science Foundation of China (21563011).

\section{REFERENCES}

[1] Jian-Yu Zhu,Jing-xia Zhang,Hui-fen Zhou,Wen-qing Qin,Li-yuan Chai, Yue-hua Hu.Microwave-assisted synthesis and characterization of ZnO-nanorod arrays[J].Trans. Nonferrous Met.Soc.China19(2009)1578-1582.

[2] Yang Lv,Min Yang,Bin Yang,Wei Liu, Hong-dan Zhang,Xu-dong Zhao,Xiao-yang Liu.Rapid Microwave--assisted Hydrothermal Synthesis of $\mathrm{SrWO}_{4}: \mathrm{Eu}^{3+}$ Nanowires and Their Luminescence Properties[J].Chem. Res. Chin. Univ., 2015, 31(2), 175-178.

[3] Qing LR. Determination of multi pesticide residues in fruit by solid phase extraction and gas chromatography with tandem mass spectrometry[J]. Chinese Journal of Health Laboratory Technology. 2012, 22(11): 2561-2565.

[4] G. Briceño,H. Schalchli,O. Rubilar,G.R. Tortella,A. Mutis,C.S. Benimeli,G. Palma,M.C. Diez. Increased diazinon hydrolysis to 2isopropyl-6-methyl-4-pyrimidinol in liquid medium by a specific Streptomyces mixed culture[J]. Chemosphere,2016.

[5] Yong-Le Peng,Xing-Li Liu,Xiao-Hong Wang,Zhi-Gang Zhao. Microwave-assisted synthesis and antibacterial activity of derivatives of 3-[1-(4-fluorobenzyl)-1 $\mathrm{H}$-indol-3-yl]-5-(4-fluorobenzylthio)-4 $\mathrm{H}$ 1,2,4-triazol-4-amine[J]. Chemical Papers,2014,683.

[6] Meiqin Shi,Lingzhi Kang,Yekun Jiang,Chun'an Ma. MicrowaveAssisted Synthesis of Mesoporous Tungsten Carbide/Carbon for Fuel Cell Applications[J]. Catalysis Letters,2014,1442.

[7] Kinga Ostrowska,Elżbieta Hejchman,Dorota Maciejewska,Agata Wodarczyk,Kamil Włojnicki,Dariusz Matosiuk,Agnieszka
Czajkowska,Izabela Młynarczuk-Biały, Łukasz Dobrzycki. Microwave-assisted preparation, structural characterization, lipophilicity, and anti-cancer assay of some hydroxycoumarin derivatives[J]. Monatshefte für Chemie - Chemical Monthly,2015, 1461.

[8] Mala Nath,Monika Vats,Parta Roy. Design and microwave-assisted synthesis of tri- and dialkyltin(IV) hippurates, characterization, in vitro anti-cancer and in vivo anti-inflammatory activities[J]. Medicinal Chemistry Research, 2015, 241.

[9] G.A. Toledo-Ibarra,K.J.G. Díaz Resendiz,H. Ventura-Ramón,F. González-Jaime,A. Vega-López,E. Becerril-Villanueva,L. Pavón,M.I. Girón-Pérez. Oxidative damage in gills and liver in Nile tilapia ( Oreochromis niloticus ) exposed to diazinon[J]. Comparative Biochemistry and Physiology, Part A, 2016.

[10] Chi-Kang Wang,Yi-Heng Shih. Facilitated ultrasonic irradiation in the degradation of diazinon insecticide[J]. Sustainable Environment Research, 2015.

[11] G.A Toledo-Ibarra,K.J.G Díaz-Resendiz,L Pavón-Romero,A.E RojasGarcía,I.M Medina-Díaz,M.I Girón-Pérez. Effects of diazinon on the lymphocytic cholinergic system of Nile tilapia fish ( Oreochromis niloticus )[J]. Veterinary Immunology and Immunopathology,2016.

[12] Saeed Hajirezaee,Ali Reza Mirvaghefi,Hamid Farahmand,Naser Agh. Effects of diazinon on adaptation to sea-water by the endangered Persian sturgeon, Acipenser persicus, fingerlings[J]. Ecotoxicology and Environmental Safety, 2016.

[13] Mayur B. Kurade,Jung Rae Kim,Sanjay P. Govindwar,Byong-Hun Jeon. Insights into microalgae mediated biodegradation of diazinon by Chlorella vulgaris : Microalgal tolerance to xenobiotic pollutants and metabolism[J]. Algal Research, 2016.

[14] Larkin D J,Tjeerdema R S. Fate and effects of diazinon.[J]. Reviews of environmental contamination and toxicology,2000,166.

[15] Lu Chensheng,Irish Rene M,Fenske Richard. Biological monitoring of diazinon exposure using saliva in an animal model.[J]. Journal of Toxicology and Environmental Health. Part A: Current Issues, 2003, 6624.

[16] Morgan Marsha K,Stout Daniel M,Jones Paul A,Barr Dana B. An observational study of the potential for human exposures to pet-borne diazinon residues following lawn applications.[J]. Environmental Research,2008,1073.

[17] Briceño G,Schalchli H,Rubilar O,Tortella G R,Mutis A,Benimeli C S,Palma G,Diez M C. Increased diazinon hydrolysis to 2-isopropyl-6methyl-4-pyrimidinol in liquid medium by a specific Streptomyces mixed culture.[J]. Chemosphere,2016,156.

[18] Hajirezaee Saeed,Mirvaghefi Ali Reza,Farahmand Hamid,Agh Naser. Effects of diazinon on adaptation to sea-water by the endangered Persian sturgeon, Acipenser persicus, fingerlings.[J]. Ecotoxicology and environmental safety, 2016, 133. 